\title{
Memory versus effector immune responses in oncolytic virotherapies
}

\author{
Cicely Macnamara, Raluca Eftimie* \\ Division of Mathematics, University of Dundee, Dundee, United Kingdom, DD1 4HN
}

\begin{abstract}
The main priority when designing cancer immuno-therapies has been to seek viable biological mechanisms that lead to permanent cancer eradication or cancer control. Understanding the delicate balance between the role of effector and memory cells on eliminating cancer cells remains an elusive problem in immunology. Here we make an initial investigation into this problem with the help of a mathematical model for oncolytic virotherapy; although the model can in fact be made general enough to be applied also to other immunological problems. Our results show that long-term cancer control is associated with a large number of persistent effector cells (irrespective of the initial peak in effector cell numbers). However, this large number of persistent effector cells is sustained by a relatively large number of memory cells. Moreover, we show that cancer control from a dormant state cannot be predicted by the size of the memory population.
\end{abstract}

Keywords: cancer modelling, effector and memory cells, tumour control, cancer dormancy

\section{Introduction}

It is well known that after successful reaction to a pathogen, long-lasting 3 immunity can be stimulated [28]. Harnessing this natural defence system,

\footnotetext{
* Corresponding author.

Phone: +44 (0)1382 384488

Fax: +44 (0)1382 385516

Email addresses: c.k.macnamara@dundee.ac.uk (Cicely Macnamara), reftimie@maths.dundee.ac.uk (Raluca Eftimie)
} 
through the use of vaccines, has long been important in the fight against infections and diseases $[3,13]$. More recently immune mechanisms have been employed to combat cancer through various immunotherapies such as virotherapies, adoptive transfer of immune cells, cytokine therapies or antibody therapies. The low success rates of these immunotherapies is mainly caused by the fact that the immune-cancer interactions are still not fully understood.

One of the emerging cancer therapies is oncolytic virotherapy, which involves both the direct action of tumour cell destruction by a virus (that usually carries tumour-associated antigens (TAAs)) and the indirect action of anti-tumour immunity (as the immune cells learn, through interaction with the virus, to recognise the TAAs) $[24,36,39]$. The interactions between the immune cells and the viruses lead to short term (or therapeutic) and long term (or prophylactic) immunity, which can be naively characterised by effector and memory immune cells, respectively [3]. In the short term effector cells act to eliminate a pathogen, while in the long-term memory cells act to prevent its reoccurrence. Memory cells are antigen-specific; they are stored after a pathogen has been eliminated [12, 25, 49] and are capable of generating new effector cells [40]. Successful cancer treatment protocols seek persistent protection against the tumour whether through permanent elimination or control.

An important research question in immunology, still unanswered at this moment, refers to whether it is effector or memory cells which play the most important role in successful treatment protocols. It has been posited that multiple treatment protocols are likely to provide better success in immune therapies. In particular, for cancer therapies, multiple and subsequent treatments provide the possibility of activating the memory cells, which can then be used to generate a stronger more targeted response against the tumour $[26,43,45,53]$. On the other hand, there is increasing evidence that longterm cancer control is accompanied by high numbers of effector cells $[4,7,35]$. Understanding the delicate balance between the anti-tumour role of effector and memory cells will improve the existent anti-cancer treatments.

Mathematical models (see, for example, [9, 15, 17, 23, 27, 33, 34, 38, $44,50,51]$ and the references therein) have shown that possible outcomes for anti-tumour therapies are: tumour elimination, tumour dormancy, tumour escape or tumour control. A distinction between dormancy and control can 
be made: tumour control occurs when the tumour is held permanently at a constant but relatively low size, while tumour dormancy is described as a prolonged period in which the tumour remains small and as such is both asymptomatic and undetectable but will at some stage grow again[37]. Although the nature of the biological mechanisms leading to tumour dormancy is not fully known [1, 42], one possible means is through tumour-immune interactions, so called immune-mediated dormancy $[16,41,46]$. It is thought that a constant interplay between the tumour and immune cells can lead to this temporary equilibrium, but eventually one population will overpower the other and either the tumour will "escape" and grow rapidly or it will be eliminated [41, 47]. Clearly, from a clinical outlook tumour escape is a negative outcome and cancer elimination is the goal of any treatment protocol. However, as we will discuss here (and as suggested before [20]), tumour control may be the only possible approach when tumour elimination is impossible. Tumour dormancy, although of short term therapeutic benefit, presents a clinical challenge in the long-term as predictions regarding its end stage (escape or elimination) may be unlikely.

In this paper, we will introduce and investigate a mathematical model for oncolytic virotherapy, which allows us to study the balance between the memory and effector immune responses that can control tumour growth or lead to tumour dormancy. Although there are many mathematical models for cancer virotherapies (see, for example, [5, 8, 18, 27, 44, 48, 52] and the references therein), the model investigated in this study is based on a more complex ODE model described in [15], which incorporated effector and memory immune responses and replicated a treatment protocol derived in [11]. In that protocol, two viruses that carried the same tumour-associated antigen (human dopachrome tautomerase, or hDCT) were administered 14 days apart. The first virus, Adenovirus (Ad), acted as a vaccine virus by provoking an immune response against the tumour antigens. As this immune response receded, memory cells were created. The second virus, Vesicular Stomatitis Virus (VSV), was an oncolytic virus. This virus not only destroyed the cancer cells directly, but provoked a much stronger immune response to the tumour antigens due to the memory cells created in the first phase. The protocol, tested on mice, did not eradicate tumours in the majority of cases but did lead to improved survival times (compared with survival times for mice treated with just one virus). The mathematical model introduced in this study focuses on the second part of this treatment protocol, 
i.e., on the oncolytic virus (injected after the formation of memory cells). Using this model, we will investigate how differences in the magnitude of the initial memory cell population lead to control, dormancy or escape of tumour cells. We will also determine the role of parameters governing the behaviour of effector cells on the outcome of the treatment.

The paper is structured as follows. In Section 2 we describe the mathematical model. In Section 3 we begin our investigation of the long-term dynamics of this model by focusing on the steady states and their stability. To get a better understanding of the balance between effector and memory immune responses, in Section 4 we discuss the steady-state behaviour of a simplified virus-free model. In fact, this simplified model is general enough to be applied to any immunotherapy and so may permit us to make stronger conclusions about the relative importance of different immune cell types in targeting cancer. In Section 5 we investigate numerically the long-term dynamics of both the full model and the simplified model paying particular attention to the effects of varying the initial memory cell population size. Finally, in Section 6 we return to the simplified model and investigate the parameters that govern the effector cells. We conclude in Section 7 with a summary and discussion of the results.

\section{Model Description}

To model the tumour-immune-virus interactions, we focus on the following populations: the uninfected $\left(x_{\mathrm{u}}\right)$ and infected $\left(x_{\mathrm{i}}\right)$ tumour cells, the memory $\left(x_{\mathrm{m}}\right)$ and effector $\left(x_{\mathrm{e}}\right)$ immune cells, and the virus particles $\left(x_{\mathrm{v}}\right)$. We assume that the virus particles are VSV particles, and that the effector/memory cells are $\mathrm{CD}^{+} \mathbf{T}$ cells. The equations below, which are adapted from [15], take into account the fact that effector cell proliferation is stimulated by both the presence of the free virus particles (as considered in [15]) and the uninfected tumour cells (an aspect not considered in [15]). Since the data in [11] ignored the spatial aspect of solid tumours, we decided to use an ODE model, with saturated interaction terms accounting for some of the tumour spatial structure. 


$$
\begin{aligned}
\frac{d x_{\mathrm{u}}}{d t} & =r x_{\mathrm{u}}\left(1-\frac{x_{\mathrm{u}}+x_{\mathrm{i}}}{k}\right)-d_{v} \frac{x_{\mathrm{u}}}{h_{u}+x_{\mathrm{u}}} x_{\mathrm{v}}-d_{u} x_{\mathrm{u}} \frac{x_{\mathrm{e}}}{h_{e}+x_{\mathrm{e}}} \\
\frac{d x_{\mathrm{i}}}{d t} & =d_{v} \frac{x_{\mathrm{u}}}{h_{u}+x_{\mathrm{u}}} x_{\mathrm{v}}-\delta x_{\mathrm{i}}-d_{u} x_{\mathrm{i}} \frac{x_{\mathrm{e}}}{h_{e}+x_{\mathrm{e}}} \\
\frac{d x_{\mathrm{m}}}{d t} & =p_{m} \frac{x_{\mathrm{v}}}{h_{v}+x_{\mathrm{v}}} x_{\mathrm{m}}\left(1-\frac{x_{\mathrm{m}}}{M}\right) \\
\frac{d x_{\mathrm{e}}}{d t} & =p_{e} \frac{x_{\mathrm{v}}+x_{\mathrm{u}}}{h_{v}+x_{\mathrm{v}}+x_{\mathrm{u}}} x_{\mathrm{m}}-d_{e} x_{\mathrm{e}}-d_{t} x_{\mathrm{u}} x_{\mathrm{e}} \\
\frac{d x_{\mathrm{v}}}{d t} & =\delta b x_{\mathrm{i}}-\omega x_{\mathrm{v}} .
\end{aligned}
$$

These equations incorporate the following biological assumptions:

- The uninfected tumour cells grow logistically at a rate $r$, up to their carrying capacity $k$. The carrying capacity is chosen specifically to correspond to the humane endpoint for experimental protocols with mice $[11,32]$ (see also Table A.2). In addition, the large carrying capacity allows us to investigate the role of oncolytic therapy on large tumours [21]. Overall, this logistic term approximates the slow-down in tumour growth dynamics, following the lack of nutrients, as observed experimentally [30]. The uninfected tumour cells are infected by the virus particles at a rate $d_{v}$, and are killed by the effector cells at a rate $d_{u}$. The saturated form of the tumour-virus interaction term accounts in part for the spatial structure of the tumour, which leads to reduced interactions between the tumour cells and viruses (studies showing that viruses usually infect only a small number of tumour cells [10]). Finally, the saturated form of the tumour-immune interaction term accounts for the reduced number of activated immune cells that reach and interact with the tumour cells [19].

- The infected tumour cells die at a rate $\delta$ (when they burst to let the replicated virus particles out). Also, they are killed by the effector cells at a rate $d_{u}$.

- The memory cells proliferate, at a rate $p_{m}$, in the presence of virus particles (virus antigens). These cells have a carrying capacity $M$, 
which models the competition for space between memory cells or competition for antigens [2]. We assume here that the memory cells persist for a very long time (compared to the effector and tumour cells), and thus we ignore their natural death rate. Parameter $h_{v}$ denotes the half-concentration of viral antigens that trigger the memory response. The saturated form of the virus-induced memory response accounts for the limited proliferation of memory cells in response to virus particles.

- The effector cells are the result of de-differentiation of memory cells in the presence of antigens (both virus antigens and tumour antigens). The de-differentiation rate is $p_{e}$. These effector cells have a natural death rate of $d_{e}$, and can be inactivated by the tumour cells at a rate $d_{t}$. For simplicity, we decided to use the same half-concentration $h_{v}$ for the antigens (both viral and tumour antigens). However, as we will discuss in Section 6, the magnitude of this parameter does not have a great influence on the dynamics.

- The virus particles are produced by the infected tumour cells at a rate $\delta b$, where $\delta$ is the death rate of infected cells and $b$ is the burst size (i.e., the number of particles inside an infected cell). Finally, these particles are eliminated by the body at a rate $\omega$.

For a more detailed description of the model, see [15]. Note that the 2-compartment model in [15] accounted for the delay in the effector immune response following virus stimulation. To gain a better understanding of the key parameters in tumour-immunevirus dynamics, in this paper we decided to ignore such a delay.

We emphasise that many of the biological processes considered in this mathematical model could have been formulated differently (see the models in $[5,8,14,15,18,27,44,48,52]$ ). For example, the proliferation of memory cells following virus stimulation was implemented differently in a previous study [15], which considered a different pathway for memory differentiation - one of the multiple pathways suggested in the literature [22]. Equally, the tumourimmune and tumour-virus interactions could have been modelled using bi-linear terms, rather than the saturated forms we give, and the tumour growth could have been modelled using a Gompertzian or exponential form $[6,27,31]$. However, it is not the goal of this 
paper to investigate the impact of the different possible descriptions of interaction terms on the outcomes of the model. Rather, it is to choose an example of interaction terms and use them to take a first look at the potential importance of effector versus memory cells during viral therapies.

\section{Steady States and Stability}

We start the investigation of model (1) by studying first its long-term dynamics. To this end, we identify all possible steady states and determine their stability. The parameter values investigated in this article (also involved in the stability of these steady states) are summarised in Appendix A. Note that these values apply to tumour-immune interactions observed in mice.

Tumour-Free Steady States (TF). The tumour-free steady states are given by $\left(0,0, x_{\mathrm{m}}^{*}, 0,0\right)$. These steady states are always unstable saddles, due to one positive eigenvalue $\lambda_{1}=r>0$. As such, this model predicts that the treatment protocol cannot lead to permanent tumour elimination. Thus, in the following, we will be concerned with investigating stable tumour-present steady states for which the tumour size is considered to be under control, i.e. below a certain threshold. For the purpose of this study, we will assume that the value of this threshold is $10^{6}$ cells (which is the initial value for the number of cancer cells $\left.x_{\mathrm{u}}(0)\right)$.

Tumour-Present, Virus-Present, Immune-Free Steady State (IF) . The single immune-free steady state is given by $\left(x_{\mathrm{u}}^{*}, x_{\mathrm{i}}^{*}, 0,0, x_{\mathrm{v}}^{*}\right)$ where

$$
x_{\mathrm{u}}^{*}=\frac{\omega h_{u}}{b d_{v}-\omega}, \quad x_{\mathrm{i}}^{*}=\frac{k-x_{\mathrm{u}}^{*}}{1+\frac{\delta k}{r x_{\mathrm{u}}^{*}}} \quad \text { and } \quad x_{\mathrm{v}}^{*}=\frac{\delta b}{\omega}\left(\frac{k-x_{\mathrm{u}}^{*}}{1+\frac{\delta k}{r x_{\mathrm{u}}^{*}}}\right) \text {. }
$$

This steady state is identical to the immune-free steady state for the model introduced in [15]. It can be easily shown (omitted here) that this state is always unstable and as such we do not consider it further.

Tumour-Present, Virus-Free Steady States (VF) . For model (1), there are multiple virus-free steady states (in fact, infinitely many). We can gain insight into these steady states by plotting the surfaces described by the righthandside of Equations (1a) and (1d) for $x_{\mathrm{m}}, x_{\mathrm{e}}$ and $x_{\mathrm{u}}$ (since $x_{\mathrm{v}}=x_{\mathrm{i}}=0$, it 
means that the remaining equations are satisfied trivially). In Figure 1 we show the intersections of these two surfaces, corresponding to the virus-free steady states of the system. Two sets of steady states satisfy these intersection curves: the tumour-free ( $\mathrm{TF}$ ) steady states (i.e., $x_{\mathrm{u}}=x_{\mathrm{e}}=0, x_{\mathrm{m}} \in \mathbb{R}$, which have already been discussed above) and the tumour-present steady states (VF), which we focus on next. We observe that for the tumour-present states, the size of the tumour ranges from low (non-zero) values, which correspond to tumour being controlled by the immune system, to very large values (the carrying capacity size, $k$ ). To achieve a low steady state tumoursize there must be sufficiently high accompanying memory and effector cell populations.

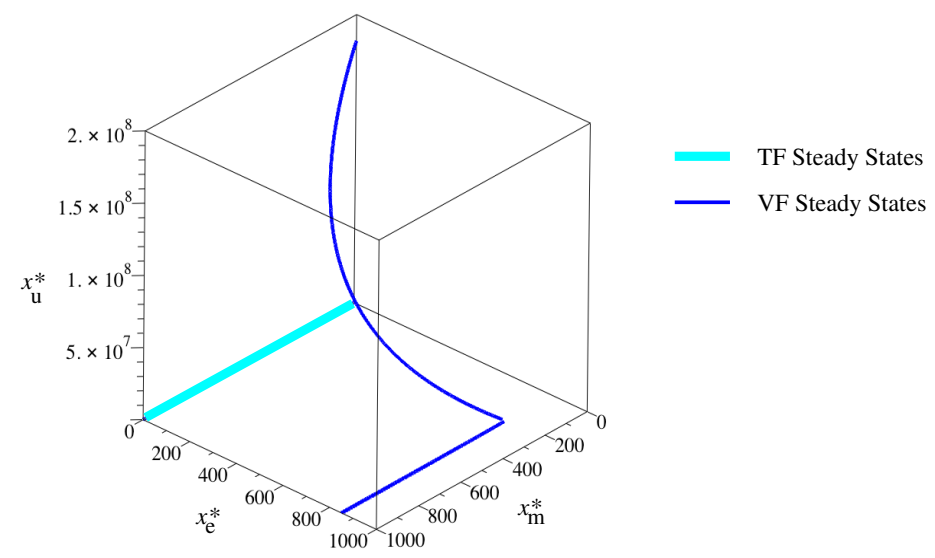

Figure 1: A plot showing the possible virus-free steady states of system (1). The tumourfree $(\mathrm{TF})$ states are given by the green thick line, the tumour-present virus-free (VF) states are given by the blue curve.

Further insight can be gleaned by considering analytic solutions to Equations (1a) and (1d). In the following we denote the steady states of $x_{\mathrm{u}}, x_{\mathrm{m}}$ and $x_{\mathrm{e}}$ by $x_{\mathrm{u}}^{*}, x_{\mathrm{m}}^{*}$ and $x_{\mathrm{e}}^{*}$, respectively. From Equation $(1 \mathrm{~d})$, we can obtain an expression for $x_{\mathrm{e}}^{*}$ in terms of $x_{\mathrm{u}}^{*}$ and $x_{\mathrm{m}}^{*}$, which is given as

$$
x_{\mathrm{e}}^{*}=\frac{p_{e} x_{\mathrm{m}}^{*} \frac{x_{\mathrm{u}}^{*}}{h_{v}+x_{\mathrm{u}}^{*}}}{d_{e}+d_{t} x_{\mathrm{u}}^{*}} .
$$


Substituting this expression into Equation (1a) and considering only the tumour-present solutions yields the cubic equation

$$
A\left(x_{\mathrm{u}}^{*}\right)^{3}+\left(B+C x_{\mathrm{m}}^{*}\right)\left(x_{\mathrm{u}}^{*}\right)^{2}+\left(D+E x_{\mathrm{m}}^{*}\right) x_{\mathrm{u}}^{*}-F=0,
$$

where

$$
\begin{gathered}
A=r d_{t} h_{e}, \quad B=r h_{e}\left(d_{e}+d_{t} h_{v}-d_{t} k\right), \quad C=r p_{e} \\
D=r h_{e}\left(d_{e} h_{v}-d_{t} h_{v} k-d_{e} k\right), \quad E=k p_{e}\left(d_{u}-r\right) \text { and } F=r k h_{e} d_{e} h_{v} .
\end{gathered}
$$

Only real positive solutions of the cubic provide biologically relevant steady states. For any given $x_{\mathrm{m}}^{*}$ we may have between one and three steady states $x_{\mathrm{u}}^{*}$.

To investigate the stability of these tumour-present virus-free steady state $\left(x_{\mathrm{u}}^{*}, 0, x_{\mathrm{m}}^{*}, x_{\mathrm{e}}^{*}, 0\right)$, we observe that the five eigenvalues of the Jacobian calculated at the steady states are: $\lambda=0$ and the two solutions of the quadratics

$$
\lambda^{2}+G_{1,2} \lambda+H_{1,2}=0,
$$

where

$$
\begin{aligned}
G_{1} & =\omega+\delta+d_{u} \frac{x_{\mathrm{e}}^{*}}{h_{e}+x_{\mathrm{e}}^{*}}, \\
H_{1} & =\omega\left(\delta+d_{u} \frac{x_{\mathrm{e}}^{*}}{h_{e}+x_{\mathrm{e}}^{*}}\right)-\delta b d_{v} \frac{x_{\mathrm{u}}^{*}}{h_{u}+x_{\mathrm{u}}^{*}},
\end{aligned}
$$

and

$$
\begin{aligned}
G_{2}= & \frac{2 r x_{\mathrm{u}}^{*}}{k}+d_{u} \frac{x_{\mathrm{e}}^{*}}{h_{e}+x_{\mathrm{e}}^{*}}-r+d_{e}+d_{t} x_{\mathrm{u}}^{*}, \\
H_{2}= & \left(d e+d_{t} x_{\mathrm{u}}^{*}\right)\left(\frac{2 r x_{\mathrm{u}}^{*}}{k}+d_{u} \frac{x_{\mathrm{e}}^{*}}{h_{e}+x_{\mathrm{e}}^{*}}-r\right) \\
& +\frac{d_{u} h_{e} x_{\mathrm{u}}^{*}}{\left(h_{e}+x_{\mathrm{e}}^{*}\right)^{2}}\left(\frac{p_{e} h_{v}}{\left(h_{v}+x_{\mathrm{u}}^{*}\right)^{2}} x_{\mathrm{m}}^{*}-d_{t} x_{\mathrm{e}}^{*}\right) .
\end{aligned}
$$

Positive eigenvalues exist, and stability fails if either $H_{1}<0$ or $H_{2}<0$ (or both). In Figure 2 we plot the states $x_{\mathrm{u}}^{*}$ against the states $x_{\mathrm{m}}^{*}$ given by the cubic (4), for the parameter values investigated in this article (see Table A.2). Here, we show also the threshold stability curves, $H_{1}=0$ and $H_{2}=0$. We note that only one branch of stable steady state solutions exists. Such states are characterised by a low (controlled) tumour size accompanied by a persistent memory cell population. 


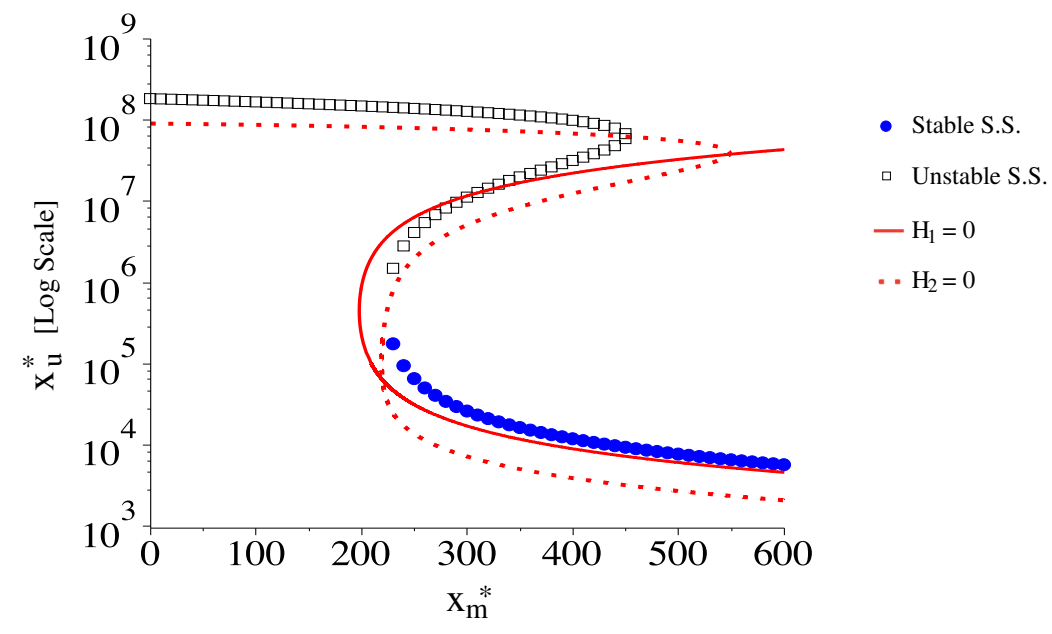

Figure 2: Plots of the steady state tumour sizes $x_{\mathrm{u}}^{*}$ against the the steady state memory size $x_{\mathrm{m}}^{*}$. To show clearly what happens for small as well as large tumour sizes, we use a $\log$ scale for $x_{\mathrm{u}}^{*}$. Stable steady states are indicated by blue circles and unstable states by black squares. We also plot the curves $H_{1}=0$ and $H_{2}=0$ in red (solid and dashed respectively), to indicate the boundaries which mark a change in stability.

Tumour-Present, Virus-Present, Immune-Present Steady State (TVI). If all populations exist, the right-hand side of Equation (1c) implies $x_{\mathrm{m}}=M$. In Figure 3 we plot the intersection curves of the surfaces given by the righthand side of Equations (1a), (1b) and (1d), in terms of the steady state populations, $x_{\mathrm{u}}^{*}, x_{\mathrm{v}}^{*}$ and $x_{\mathrm{e}}^{*}$ (using $x_{\mathrm{m}}=M$ and replacing $x_{\mathrm{i}}$ with $\omega x_{\mathrm{v}}^{*} / \delta b$, determined from the right-hand side of Equation (1e)). We observe that there are only two distinct biologically relevant intersections of all three surfaces corresponding to steady states of model (1). Neither of these states, namely the TF steady state $\left(x_{\mathrm{u}}=x_{\mathrm{v}}=0\right)$ and a VF steady state $\left(x_{\mathrm{u}} \approx 221\right.$, $x_{\mathrm{m}}=M=10^{4}, x_{\mathrm{e}} \approx 864$ ), has all five populations present. Thus, at least for the parameter values investigated in this article (see Appendix A), a TVI state does not exist and as such we should concern ourselves with stabilising, at a low tumour size, the virus-free (VF) steady states discussed previously. Biologically, our concern with this VF state makes sense, as we would hope to find a treatment protocol in which, after reducing the tumour size, the virus would be cleared. 


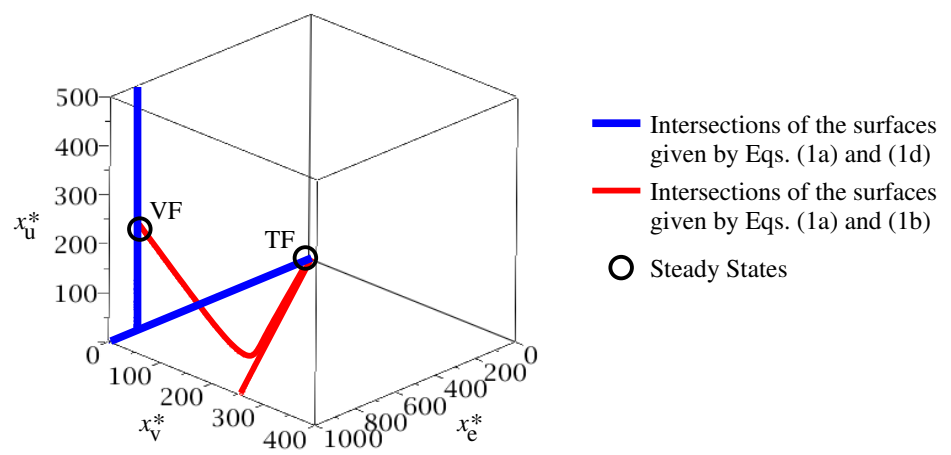

Figure 3: A plot showing the intersections of surfaces described by equations (1a), (1b) and (1d). Circled are the possible steady states for model (1), when $x_{\mathrm{m}}=M$.

\section{A simplified virus-free system}

Next, we consider a completely virus-free system. We will return to this model in the next sections, when we will investigate the role of the memory versus effector immune responses in tumour control. In the absence of the virus, system (1) reduces to

$$
\begin{aligned}
\frac{d x_{\mathrm{u}}}{d t} & =r x_{\mathrm{u}}\left(1-\frac{x_{\mathrm{u}}}{k}\right)-d_{u} x_{\mathrm{u}} \frac{x_{\mathrm{e}}}{h_{e}+x_{\mathrm{e}}}, \\
\frac{d x_{\mathrm{m}}}{d t} & =0 \\
\frac{d x_{\mathrm{e}}}{d t} & =p_{e} \frac{x_{\mathrm{u}}}{h_{v}+x_{\mathrm{u}}} x_{m}-d_{e} x_{\mathrm{e}}-d_{t} x_{\mathrm{u}} x_{\mathrm{e}} .
\end{aligned}
$$

The steady states $\left(x_{\mathrm{u}}^{*}, x_{\mathrm{m}}^{*}, x_{\mathrm{e}}^{*}\right)$ of this system still satisfy equations (3) and (4). We note from equation (9b) that the memory cell population does not change and as such will remain at its initial size. Thus, we may consider $x_{\mathrm{m}}^{*}=x_{\mathrm{m}}(0)$. Therefore, the solutions for $x_{\mathrm{u}}^{*}$ obtained by solving (4) depend directly on the initial memory cell population size.

The eigenvalues of system (9) are governed by

$$
\lambda\left(\lambda^{2}+G_{2} \lambda+H_{2}\right)=0,
$$

where $G_{2}$ and $H_{2}$ are given as before. Thus, stability of the virus-free system is governed solely by the sign of $H_{2}$. In Figure 4 we plot the steady state 
tumour sizes, $x_{\mathrm{u}}^{*}$, against the steady state memory size, $x_{\mathrm{m}}^{*}$ (as in Figure 2, but now with only the $H_{2}=0$ stability boundary). We observe that for a range of $x_{\mathrm{m}}^{*}$ values $\left(x_{\mathrm{m}}^{*} \approx 230-460\right)$ the system is bistable. However, investigation of the long-term behaviour of system (9) shows that the system always chooses one stable steady state (filled blue circles in Figure 4(a)). We observe that the transition from the upper stable branch to the lower stable branch occurs as the maximum tumour size crosses the unstable branch (described by black squares). Hence, the unstable branch of steady states $x_{\mathrm{u}}^{*}$ acts as a separatrix: if the solution for $x_{\mathrm{u}}$ reaches any point above this branch the dynamics will approach the upper stable steady state; on the other hand, if the solution remains below this branch, the dynamics will approach the lower stable steady state. To indicate this, we also include the maximum tumour sizes attained for each $x_{\mathrm{m}}(0)=x_{\mathrm{m}}^{*}$ in Figure 4 (see red crosses).

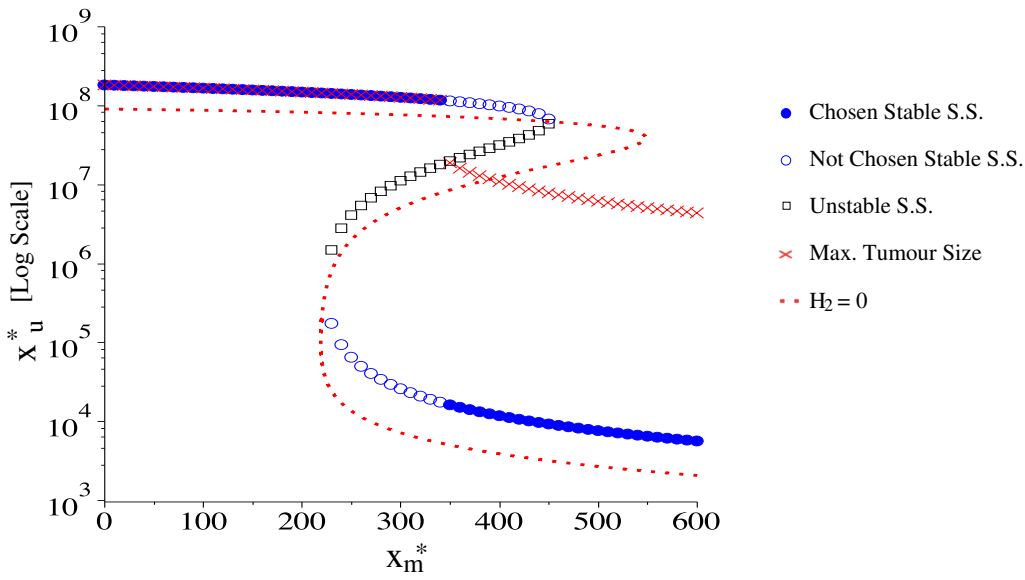

Figure 4: Plot of the steady state tumour sizes $x_{\mathrm{u}}^{*}$ against the the steady state memory sizes $x_{\mathrm{m}}^{*}=x_{\mathrm{m}}(0)$. To show more clearly what happens for small and large tumour sizes, we use a $\log$ scale for $x_{\mathrm{u}}^{*}$. Stable steady states are indicated by blue circles and unstable by black squares. The dynamics of the system evolves towards the filled blue circles. We also include the curve $H_{2}=0$ (red dashed curve) to indicate the boundary which marks a change in stability and the maximum tumour size (red crosses) attained for each $x_{\mathrm{m}}(0)=x_{\mathrm{m}}^{*}$. 
(a)

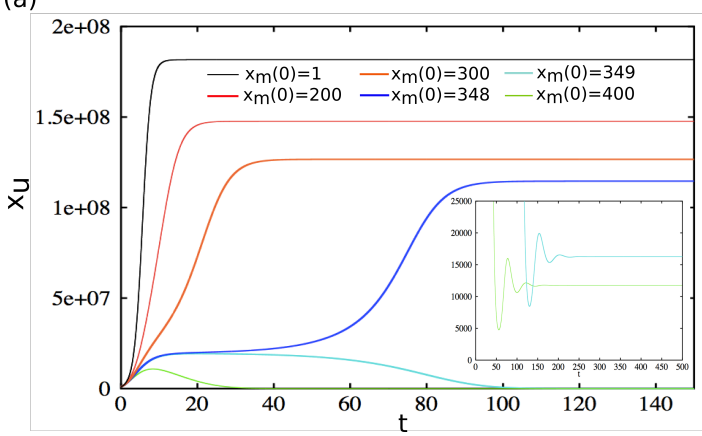

\section{Tumour growth dynamics}

(b)

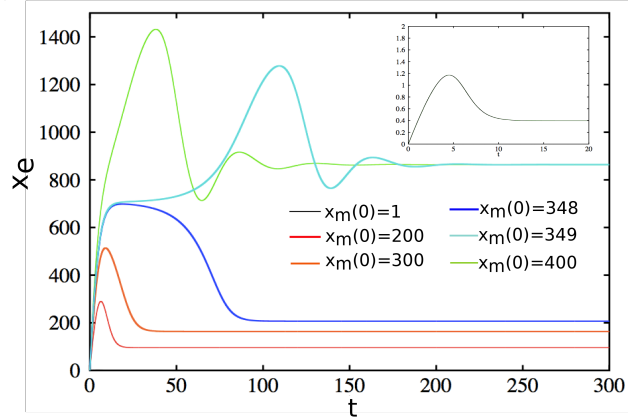

Figure 5: Explicit time plots for (a) the uninfected tumour size, (b) the effector population for different values of the initial memory cell-population for virus-free initial conditions. In each case $x_{\mathrm{u}}(0)=10^{6}$ and $x_{\mathrm{v}}(0)=x_{\mathrm{i}}(0)=x_{\mathrm{e}}(0)=0$. All parameters are as in Table A.2.

In this section, we investigate the time-evolution of systems (9) and (1) towards the VF steady states described previously.

cos $x_{\mathrm{u}}(0)=10^{6}$ and $x_{\mathrm{v}}(0)=x_{\mathrm{T}}(0)=x_{\mathrm{e}}(0)=0$. All parameters are as in Table A.2.

We start by discussing first the dynamics of the virus-free system (9). In Figure 5 we plot: (a) the explicit time behaviour of the tumour population, and (b) the explicit time behaviour of the effector population, for different values of the initial memory cell population $x_{\mathrm{m}}(0)$. (The initial conditions for the other variables are $x_{\mathrm{u}}(0)=10^{6}, x_{\mathrm{e}}(0)=x_{\mathrm{v}}(0)=x_{\mathrm{i}}(0)=0$.) This plot corresponds directly with the behaviour predicted by Figure 4: increasing the initial memory cell population leads to a lower steady state for the tumour size and a higher steady state for the effector population size. A substantial jump in tumour/effector size occurs between $x_{m}(0)=348$ and $x_{\mathrm{m}}(0)=349$. When $x_{\mathrm{m}}(0)=348$ we observe a period of cancer dormancy (corresponding to a sustained "high" effector population size), between $t=10$ and $t=60$ days. However, the tumour begins to grow again and achieves a high steadystate size. When $x_{\mathrm{m}}(0)=349$ the system appears similarly dormant, but then tends to a much lower steady-state tumour size (low enough to be considered under control). When the steady state for the tumour population is on the lower branch of the stable solutions shown in Figure 4, the effector population always tends towards the steady state $x_{\mathrm{e}}^{*} \approx 864$ cells.

Note that the behaviour shown in Figure 5 is for initial conditions with 
(a)

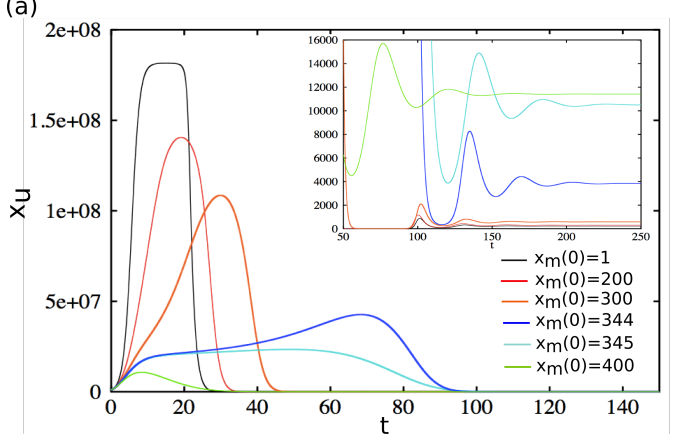
value of $x_{\mathrm{u}}^{*}$. (b)

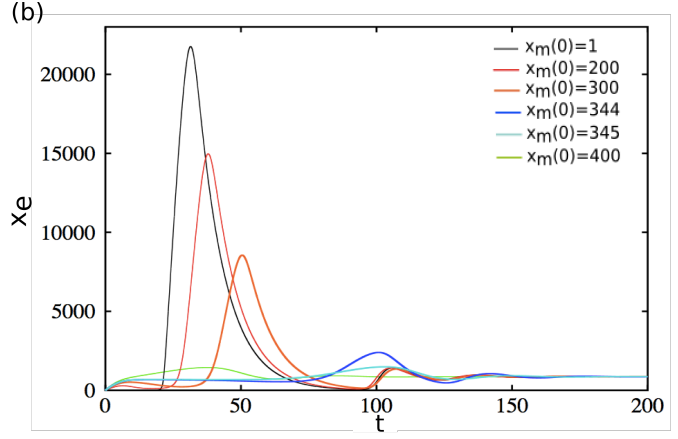

Figure 6: Explicit time plots for (a) the uninfected tumour size, (b) the effector population for different values of the initial memory cell-population for virus-present initial conditions. In each case $x_{\mathrm{u}}(0)=10^{6}, x_{\mathrm{v}}(0)=1$ and $x_{\mathrm{i}}(0)=x_{\mathrm{e}}(0)=0$. All parameters are as in

Table A.2.

zero effector cells $\left(x_{\mathrm{e}}(0)=0\right)$. If we add an initial effector cell population to the system, it has the effect of slightly reducing the value of $x_{\mathrm{m}}(0)$ for which we achieve the jump to the lower steady-state branch for the tumour cells. For example, if $x_{\mathrm{e}}(0)=100$ we require $x_{\mathrm{m}}(0) \geq 323$ to achieve the lower

We next consider the behaviour of the full system (1) (which is not virusfree, but evolves towards a virus-free steady state over time), as we vary the initial memory cell population. In Figure 6 we plot (a) the explicit time behaviour of the tumour population, and (b) the explicit time behaviour of the effector population, for different values of the initial memory cell population $x_{\mathrm{m}}(0)$. In Figure 6(a) we observe that introducing a single virus particle reduces the tumour size to a low and controlled steady state, for all values of $x_{\mathrm{m}}(0)$. Figure 2 predicted that only low tumour sizes for VF states were stable. Note that for low $x_{\mathrm{m}}(0)$ values, the tumour first grows towards a very large "fatal" size, before decaying to a low steady-state value. Thus, as we vary $x_{\mathrm{m}}(0)$, it becomes important to consider not only the steady state tumour size but also the maximum tumour size. Figure 6(b) shows that in each case the effector population tends to $x_{\mathrm{e}}^{*} \approx 864$.

Unexpected dynamics can be seen in the inset to Figure 6(a): increasing $x_{\mathrm{m}}(0)$ leads to an increase in the steady-state $x_{\mathrm{u}}^{*}$. To get a better understanding of why this happens, in Figure 7 we plot both the maximum tumour 
size and the steady-state tumour size against the initial memory population size $x_{\mathrm{m}}(0)$, for $x_{\mathrm{v}}(0)=\left\{1,10^{2}, 10^{4}, 10^{6}\right\}$. As observed in Figure $7(\mathrm{a})$, when we introduce one virus particle, a low initial memory population gives rise to a low steady-state tumour size. However, this behaviour is also accompanied by a higher peak in the tumour size. As we increase $x_{\mathrm{m}}(0)$, the maximum tumour size decreases while the steady-state value for the tumour increases. This increasing/decreasing behaviour becomes particularly strong for $x_{\mathrm{m}}(0) \in(340,350)$. Note that for $x_{\mathrm{m}}(0)>430$, the maximum tumour sizes and the steady-state tumour sizes are below the thresholds of $10^{7}$ and $10^{6}$ cells, respectively. These thresholds are sufficiently low to ensure the survival of the mice. In Figure $7(\mathrm{~b})$ (where $x_{\mathrm{v}}(0)=10^{2}$ ), the sharp changes in both the maximum tumour size and the steady-state size are no longer observed. Instead both profiles are continuous and the steady-state size achieves a much lower peak. As we increase the initial virus population further (see Figure $7(\mathrm{c})$ ), the maximum tumour size reduces more rapidly, while the steady-state tumour size remains almost constant at $x_{\mathrm{u}}^{*} \approx 221$ cells, far below the threshold of $10^{6}$ cells. We do note, however, that even when $x_{\mathrm{v}}(0)=10^{6}$ and there is a high initial memory population size (see Figure $7(\mathrm{~d})$ ), the peak of the tumour size is above $10^{6}$ cells (although the attained size is short-lived and not typically fatal).

\section{Memory versus immune responses on tumour growth}

To compare the importance of the memory versus immune responses in tumour elimination, we focus on the simplified virus-free system. In Figure 8, we graph the steady states $x_{\mathrm{u}}^{*}$ against the steady states $x_{\mathrm{m}}^{*}$ (equivalent to the initial memory population in this case) along with the stability boundary $H_{2}=0$, while changing different parameters that control the effector immune response. In Figure 8(a) we change the rate $p_{e}$ that controls the dedifferentiation of memory cells into effector cells. Increasing this rate reduces the required initial memory size to achieve a lower steady-state tumour size. Decreasing the the natural effector decay rate $d_{e}$ (see Figure $8(\mathrm{~b})$ ) also leads to a reduction in the initial memory size required to achieve a lower steadystate tumour size. Similar results are obtained when decreasing the effector half-saturation constant $h_{e}$ (see Figure 8(c)). In Figure 8(d) we include a plot which shows the effect of changing $h_{v}$. We see here that there are almost no differences in the long-term behaviour of system (1) when $1<h_{v}<10^{4}$. However, for $h_{v}=10^{6}$ there are small differences in the size of $x_{\mathrm{u}}^{*}$ approached 

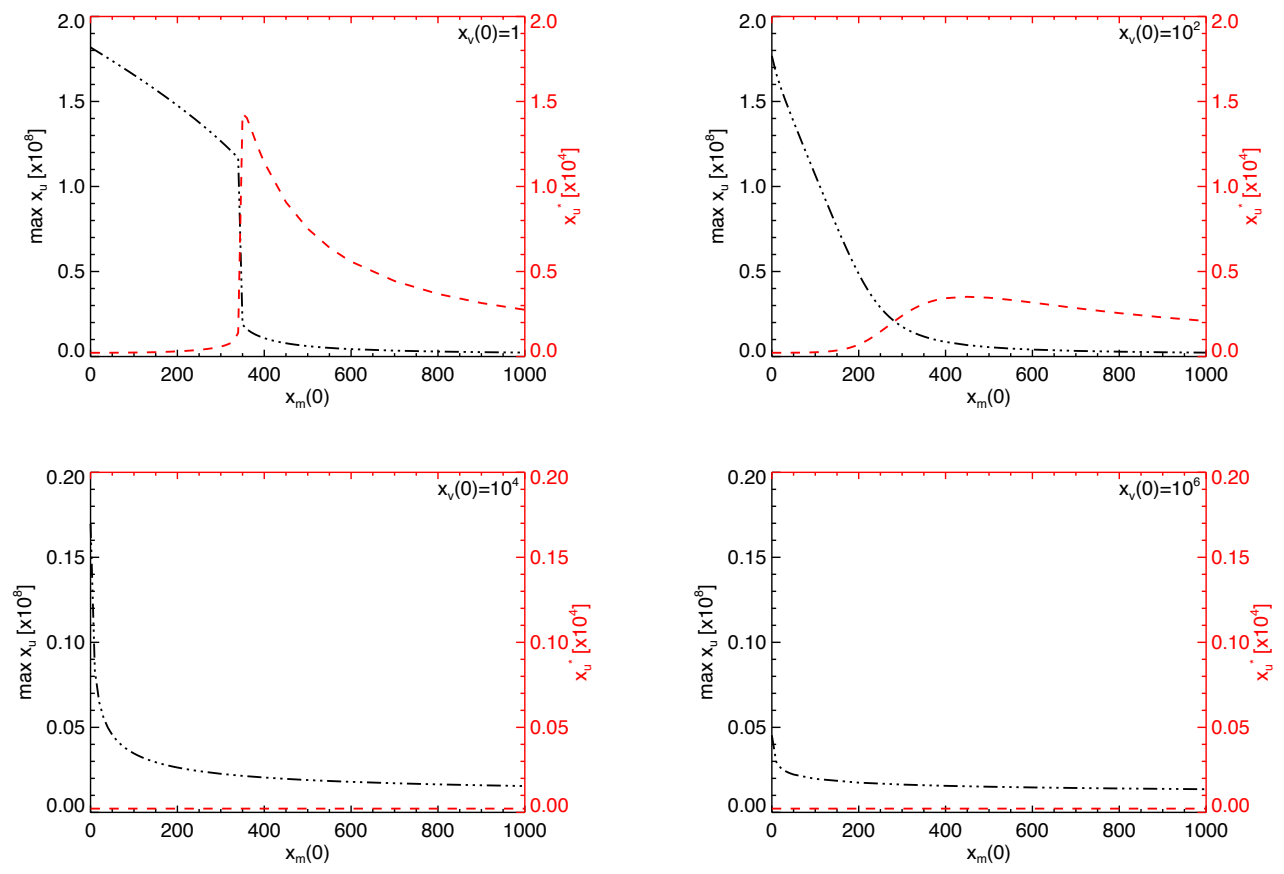

Figure 7: Plots of the maximum tumour size (black dashed-dot curve) and the steady-state tumour size (red dashed curve), for different values of the initial virus population. The parameter values are as in Table A.2.

by system (1), for initial memory population sizes $x_{\mathrm{m}}^{*} \in(200,450)$.

We observed above (see Figures 5 for the virus-free system and Figure 6 for the virus-present system) that a low steady-state tumour size was accompanied by an effector steady-state size of $x_{\mathrm{e}}^{*} \approx 864$ cells. To achieve this steady-state effector population size we must either have a high enough initial memory population or, as shown in Figure 8, be able to control immunerelated parameters i.e., provoke a higher de-differentiation of memory cells to effector cells, reduce the effector cell natural decay and enhance effectortumour interactions. However, it might not be possible to control these parameters experimentally. And even if we can alter them favourably, a higher initial memory population size continues to be important. As such, we conclude that focus should remain on stimulating a high initial memory population. 

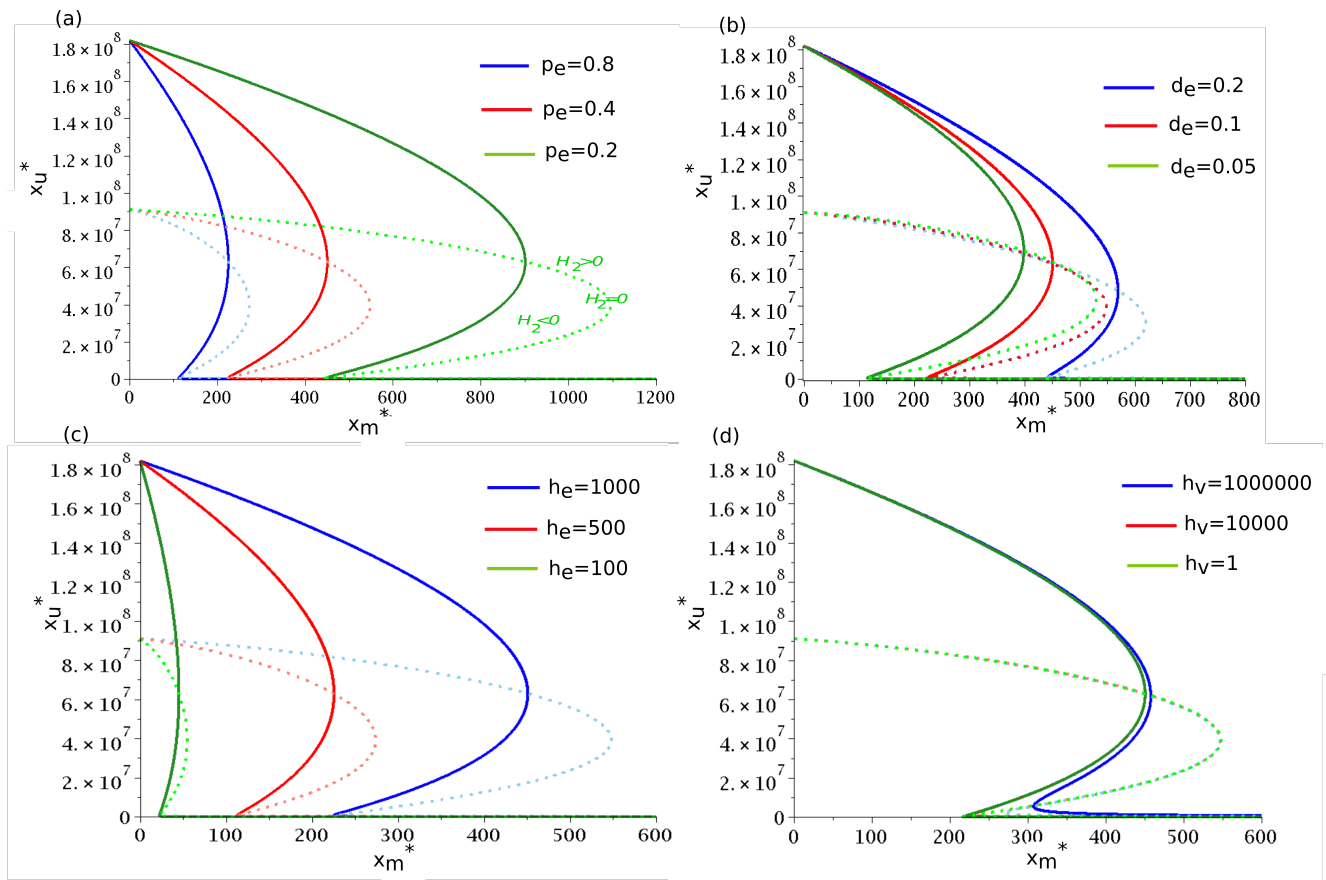

Figure 8: Plots showing the steady state behaviour (solid curves) of the virus-free system along with stability boundaries $H_{2}=0$ (dashed curves) for different parameters. In panel (a) we change $p_{e}$, in panel (b) we change $d_{e}$, in panel (c) we change $h_{e}$ and in panel (d) we change $h_{v}$, while keeping all other parameters fixed as in Table A.2.

\section{Discussion}

In this article, we introduced a simple, nonlinear mathematical model that described the interactions among immune cells, cancer cells and viruses. Although the original purpose of the model was to investigate the dynamics of oncolytic therapy, much of what we have shown applies to a model of a virus-free system. As such, the model could be used to give insight into immune-cancer interactions after the stimulation of anti-cancer immune memory cells. We focussed our attention on the importance of memory and effector cell population sizes on stabilising the tumour-present virus-free steady states.

We found that for our model (system (1)) the dynamic behaviour always evolved towards a tumour-present virus-free steady state, whether under virus-free or virus-present initial conditions. When the system was fully 
virus-free, we found that only by increasing the initial memory cell population could we achieve reduced tumour growth and low steady-state tumour size. For the virus-present system, it was important to have a high initial memory cell population in order to reduce the initial growth (and maximum size) of the tumour (although there was a slight trade-off as the steady-state size increased as we increased $\left.x_{\mathrm{m}}(0)\right)$. Having a high initial memory cell population became less important as we increased the initial virus population, $x_{\mathrm{v}}(0)$. Indeed when $x_{\mathrm{v}}(0)=10^{6}$ there was very little difference in the maximum tumour size and no difference in the steady-state tumour size, for all values of $x_{\mathrm{m}}(0)$. A parameter investigation showed that provoking a high initial memory population would always lead to a positive outcome, and that biologically this is likely to be more attainable than stimulating changes to the immune-related parameters. Importantly, we have found that low steady-state tumour sizes were always accompanied by a high steady state effector population (always around $x_{\mathrm{e}}^{*} \approx 864$ cells). As such, this adds to the evidence that suggests that cancer control is the result of a persisting population of effector cells, regardless of the initial number immune cells $[4,35,7]$.

Our investigations also indicated that specific conditions could lead to immune-mediated cancer dormancy. It is now evident from the literature that cancers may remain dormant for prolonged periods of time, after which tumours will either escape (and grow excessively) or be eliminated. Here, we showed that very slight changes to the system set-up (in our case slight changes to the initial memory cell population) could lead to a change between these two contrasting outcomes. Furthermore, with a wide range of parameters it is unlikely that we could predict whether the patient would go on to experience cancer growth or cancer reduction and control after dormancy. Unfortunately due to the very nature of cancer dormancy (i.e., cancer is at a very small size), it is often elusive to the methods of detection currently available to clinicians. Our findings remind us that it remains of great importance to search for ways to detect and monitor cancer dormancy.

Having discussed our results, we wish to stress that they are subject to the limitations of our model. As mentioned, when discussing the model set up, there are alternative ways of incorporating the biological mechanisms known to occur, and equally we have only attempted to describe certain biological pathways which are still not fully understood. Different formulations of the model could 
well provide further insight into the role of effector and memory cells; in fact the subject may benefit from a detailed investigation of general interaction terms. However, our investigation into this important immunological problem aims to be a starting point for further discussion on this topic.

\section{Acknowledgements}

RE acknowledges support from an Engineering and Physical Sciences Research Council (UK) First Grant number EP/K033689/1.

\section{Appendix A. Appendices}

\begin{tabular}{cll}
\hline Variables & Meaning & Initial Value \\
\hline$x_{\mathrm{u}}$ & uninfected cancer cells & $10^{6}$ \\
$x_{\mathrm{i}}$ & infected cancer cells & 0 \\
$x_{\mathrm{m}}$ & memory cells & $1-10^{4}$ \\
$x_{\mathrm{e}}$ & effector cells & 0 \\
$x_{\mathrm{v}}$ & virus particles & $0-10^{6}$ \\
\hline
\end{tabular}

Table A.1: Initial values of the variables for the model given by Equations (1a)-(1e).

\section{References}

[1] Almog, N., 2010. Molecular mechanisms underlying tumor dormancy. Cancer Lett. 294 (2), 139-46.

[2] Antia, R., Pilyugin, S., Ahmed, R., 1998. Models of immune memory: On the role of cross-reactive stimulation, competition, and homeostasis in maintaining immune memory. Proc. Natl. Acad. Sci. USA.

[3] Bachmann, M. F., Jennings, G. T., Nov 2010. Vaccine delivery: a matter of size, geometry, kinetics and molecular patterns. Nat. Rev. Immunol. 10 (11), 787-96.

[4] Baitsch, L., Baumgaertner, P., Devêvre, E., Raghav, S. K., Legat, A., Barba, L., Wieckowski, S., Bouzourene, H., Deplancke, B., Romero, P., Rufer, N., Speiser, D. E., Jun 2011. Exhaustion of tumor-specific cd8 $\mathrm{t}$ cells in metastases from melanoma patients. J. Clin. Invest. 121 (6), 2350-60. 


\begin{tabular}{|c|c|c|c|}
\hline Parameter & Value & Units & Description \& Reference \\
\hline$r$ & 0.927 & days $^{-1}$ & proliferation rate for tumour cells [11] \\
\hline$k$ & $1.8182 \times 10^{8}$ & cells/vol & carrying capacity for the tumour cells \\
\hline$d_{\mathrm{v}}$ & 0.0038 & $($ cells $/$ vol $)(\mathrm{PFU} / \mathrm{vol})^{-1}(\text { days })^{-1}$ & $\begin{array}{l}\text { infection rate of tumour cells with the oncolytic } \\
\text { virus }\end{array}$ \\
\hline$d_{\mathrm{u}}$ & 2.0 & days $^{-1}$ & $\begin{array}{l}\text { lysis rate of tumour cells (infected and uninfec- } \\
\text { ted) by the immune cells [29] }\end{array}$ \\
\hline$h_{\mathrm{u}}$ & 1 & cells/vol & $\begin{array}{l}\text { half-saturation constant for the tumour cells in- } \\
\text { fected with the oncolytic virus }\end{array}$ \\
\hline$h_{\mathrm{e}}$ & $10^{3}$ & cells/vol & $\begin{array}{l}\text { half-saturation constant for the effector cells } \\
\text { that support half the maximum killing rate }\end{array}$ \\
\hline$h_{\mathrm{v}}$ & $10^{4}$ & $\mathrm{PFU} / \mathrm{vol}$ & $\begin{array}{l}\text { half-saturation constant of (viral \& tumour) } \\
\text { antigens that induce half the maximum pro- } \\
\text { liferation rate of immune cells }\end{array}$ \\
\hline$\delta$ & 1 & days $^{-1}$ & $\begin{array}{l}\text { rate at which the oncolytic virus kills the tumor } \\
\text { cells }\end{array}$ \\
\hline$p_{\mathrm{m}}$ & 2.5 & days $^{-1}$ & $\begin{array}{l}\text { proliferation rate of memory cells following sec- } \\
\text { ondary encounter with tumor antigens carried } \\
\text { by virus particles [11] }\end{array}$ \\
\hline$M$ & $10^{4}$ & $($ cells $) / \mathrm{vol}$ & carrying capacity for memory cells \\
\hline$p_{\mathrm{e}}$ & 0.4 & days $^{-1}$ & $\begin{array}{l}\text { rate at which memory cells become effector } \\
\text { cells following secondary encounter with tumor } \\
\text { antigens carried by virus particles }\end{array}$ \\
\hline$d_{\mathrm{e}}$ & 0.1 & days $^{-1}$ & death rate of effector cells [11] \\
\hline$d_{\mathrm{t}}$ & $5 \times 10^{-9}$ & $(\text { cells })^{-1}($ vol $)(\text { days })^{-1}$ & $\begin{array}{l}\text { inactivation rate of immune effector cells by the } \\
\text { tumor cells }\end{array}$ \\
\hline$\omega$ & 2.042 & days $^{-1}$ & $\begin{array}{l}\text { decay rate for the concentration of oncolytic } \\
\text { virus (VSV) particles in the blood }\end{array}$ \\
\hline$b$ & 1000 & $(\mathrm{PFU} / \mathrm{vol})(\mathrm{cell})^{-1}(\mathrm{vol})$ & $\begin{array}{l}\text { number of virus (VSV) particles released from } \\
\text { an infected cell, capable of forming plaques }\end{array}$ \\
\hline
\end{tabular}

Table A.2: Parameters of model used throughout and values used for numerical simulations. In each case they are as given in [15] (or at least within the ranges given). Throughout this report, we consider the density of cells (i.e., cell numbers per blood volume (vol)) and the plaque-forming units (PFU) for the virus particles. (PFU is a generally accepted functional measurement for the virus particles; defective viruses which do not form a plaque cannot infect their target and are discounted.)

[5] Bajzer, Z., Carr, T., Josić, K., Russell, S., Dingli, D., 2008. Modeling of cancer virotherapy with recombinant measles viruses. J. Theor. Biol. $252,109-122$.

[6] Benzekry, S., Lamont, C., Beheshti, A., Tracz, A., Ebos, J. M. L., Hlatky, L., Hahnfeldt, P., Aug 2014. Classical mathematical models for description and prediction of experimental tumor growth. PLoS Comput Biol 10 (8), e1003800.

[7] Berezhnoy, A., Rajagopalan, A., Gilboa, E., 2014. A clinically useful 
approach to enhance immunological memory and antitumor immunity. OncoImmunology 3, e28811.

[8] Biesecker, M., Kimn, J.-H., Lu, H., Dingli, D., Bajzer, Z., 2010. Optimization of virotherapy for cancer. Bull Math Biol 72 (2), 469-89.

[9] Bozic, I., Allen, B., Nowak, M. A., 2012. Dynamics of targeted cancer therapy. Trends Mol. Med. 18, 311-316.

[10] Breitbach, C., Paterson, J., Lemay, C., Falls, T., McGuire, A., Parato, K., Stojdl, D., Daneshmand, M., Speth, K., Kirn, D., McCart, J., Atkins, H., Bell, J., 2007. Targeted inflammation during oncolytic virus therapy severely compromises tumor blood flow. Molecular Therapy $15(9), 1686-93$.

[11] Bridle, B., Stephenson, K., Boudreau, J., Koshy, S., Kazdhan, N., Pullenayegum, E., Brunellière, J., Bramson, J., Lichty, B., Wan, Y., 2010. Potentiating cancer immunotherapy using an oncolytic virus. Mol. Ther. 18, 1430-1439.

[12] Crotty, S., Ahmed, R., 2004. Immunological memory in humans. Semin. Immunol. 16, 197-203.

[13] Dermime, S., Armstrong, A., Hawkins, R., Stern, P., 2002. Cancer vaccines and immunotherapy. Brit. Med. Bull. 62, 149-162.

[14] Eftimie, R., Bramson, J. L., Earn, D. J. D., 2011. Interactions between the immune system and cancer: a brief review of non-spatial mathematical models. Bull Math Biol 73 (1), 2-32.

[15] Eftimie, R., Dushoff, J., Bridle, B., Bramson, J., Earn, D., 2011. Multistability and multi-instability phenomena in a mathematical model of tumor-immune-virus interactions. Bull. Math. Biol. 73 (12), 2932-2961.

[16] Farrar, J., Katz, K., Windsor, J., Thrush, G., Scheuermann, R., Uhr, J., Street, N., 1999. Cancer dormancy. VII. A regulatory role for CD8 ${ }^{+}$ $\mathrm{T}$ cells and IFN- $\gamma$ in establishing and maintaining the tumour-dormant state. J. Immunol. 162, 2842-2849.

[17] Ferreira Jr., S. C., Martinsa, M. L., Vilela, M. J., 2005. Fighting cancer with viruses. Physica A 345, 591-602. 
[18] Friedman, A., Tian, J., Fulci, G., Chiocca, E., Wang, J., 2006. Glioma virotherapy: effects of innate immune suppression and increased viral replication capacity. Cancer Res. 66 (4), 2314-2319.

[19] Gajewski, T., Schreiber, H., Fu, Y.-X., 2013. Innate and adaptive immune cells in the tumor microenvironment. Nature Immunology 14 (10), $1014-22$.

[20] Gatenby, R., 2009. A change of strategy in the war on cancer. Nature 459, 509-509.

[21] Ikeda, K., Ichikawa, T., Wakimoto, H., Silver, J., Deisboeck, T., Finkelstein, D., Harsh IV, G., Louis, D., Bartus, R., Hochberg, F., Chiocca, E., 1999. Oncolytic virus therapy of multiple tumors in the brain requires suppression of innate and elicited antiviral responses. Nature Medicine $5(8), 881-887$.

[22] Kaech, S., Wherry, J., Ahmed, R., 2002. Effector and memory t-cell differentiation: implications for vaccine development. Nature Reviews Immunology 2, 251-262.

[23] Karev, G. P., Novozhilov, A. S., Koonin, E. V., 2006. Mathematical modeling of tumor therapy with oncolytic viruses: effects of parametric heterogeneity on cell dynamics. Biol. Direct. 1, 30.

[24] Kelly, E., Russell, S. J., 2007. History of oncolytic viruses: Genesis to genetic engineering. Mol. Ther. 15, 651-659.

[25] Klebanoff, C. A., Gattinoni, L., Restifo, N. P., 2006. CD8+ T-cell memory in tumor immunology and immunotherapy. Immunol. Rev. 211, $214-224$.

[26] Klebanoff, C. A., Gattinoni, L., Torabi-Parizi, P., Kerstann, K., Cardones, A. R., Finkelstein, S. E., Palmer, D. C., Antony, P. A., Hwang, S. T., Rosenberg, S. A., Waldmann, T. A., Restifo, N. P., 2005. Central memory self tumor-reactive CD8+ T cells confer superior antitumor immunity compared with effector memory T cells. PNAS 102, 9571-9576.

[27] Komarova, N. L., Wodarz, D., 2010. Ode models for oncolytic virus dynamics. J. Theor. Biol. 263 (4), 530-43. 
[28] Kumar, H., Kawai, T., Akira, S., 2011. Pathogen recognition by the innate immune system. Int. Rev. Immunol. 30 (1), 16-34.

[29] Kündig, T., Bachmann, M., Oehen, S., Hoffmann, U., Simard, J., Kalberer, C., Pircher, H., Ohashi, P., Hengartner, H., Zinkernagel, R., 1996. On the role of antigen maintaining cytotoxic T-cell memory. Proc. Natl. Acad. Sci. USA 93, 9716-9723.

[30] Laird, A., 1964. Dynamics of tumor growth. Br. J. Cancer 18, 490-502.

[31] Marusić, M., M., Vuk-Pavlovic, S., 1993. Prediction power of mathematical models for tumour growth. Journal of Biological Systems 1 (1), 69-78.

[32] N.I.H., O.A.C.U., 1996. Guidelines for endpoints in animal study proposals. http://oacu.od.nih.gov/ARAC/documents/ASP_Endpoints.pdf.

[33] Paiva, L. R., Binny, C., Ferreira, Jr., S. C., Martins, M. L., February 2009. A multiscale mathematical model for oncolytic virotherapy. Cancer Res. 69.

[34] Paiva, L. R., Martins, M. L., Ferreira, Jr., S. C., 2011. Questing for an optimal, universal viral agent for oncolytic virotherapy. Physical Review E 84 .

[35] Paulis, L., Mandal, S., Kreutz, M., Figdor, C., 2013. Dendritic cell-based nanovaccines for cancer immunotherapy. Curr. Opp. Immunol. 25, 389395.

[36] Pol, J. G., Rességuier, J., Lichty, B. D., 2012. Oncolytic viruses: a step into cancer immunotherapy. Virus Adapt. and Treat. 4, 1-21.

[37] Quesnel, B., 2008. Dormant tumor cells as therapeutic target? Cancer Lett. 267, 10-17.

[38] Rommelfanger, D. M., Offord, C. P., Dev, J., Bajzer, Z., Vile, R. G., Dingli, D., 2012. Dynamics of melanoma tumor therapy with vesicular stomatitis virus: explaining the variability in outcomes using mathematical modeling. Gene. Ther. 19 (5), 543-9.

[39] Russell, S. J., Peng, K.-W., Bell, J. C., 2012. Oncolytic virotherapy. Nat. Biotechnol. 30 (7), 658-70. 
[40] Sallusto, F., Geginat, J., Lanzavecchia, A., 2004. Central memory and effector memory t cell subsets: function, generation, and maintenance. Annu. Rev. Immunol. 22, 745-763.

[41] Teng, M., Swann, J., Koebel, C., Schreiber, R., Smyth, M., 2008. Immune-mediated dormancy: an equilibrium with cancer. J. Leukoc. Biol. 84, 988-993.

[42] Uhr, J., Pantel, K., 2011. Controversies in clinical cancer dormancy. Proc. Natl. Acad. Sci. USA 108, 12396-12400.

[43] van Duikeren, S., Fransen, M. F., Redeker, A., Wieles, B., Platenburg, G., Krebber, W.-J., Ossendorp, F., Melief, C. J. M., Arens, R., 2012. Vaccine-induced effector-memory cd8+ t cell responses predict therapeutic efficacy against tumors. J. Immunol. 189 (7), 3397-403.

[44] Wein, L. M., Wu, J. T., Kirn, D., 2003. Validation and analysis of a mathematical model of a replication-competent oncolytic virus for cancer treatment: Implications for virus design and delivery. Cancer Res. 63, 1317-1324.

[45] Wherry, J. E., Ahmed, R., 2004. Memory cd8 t-cell differentiation during viral infection. J. Virol. 78 (11), 5535-45.

[46] Wilkie, K., Hahnfeldt, P., 2013. Mathematical models of immuneinduced cancer dormancy and the emergence of immune evasion. Interface Focus 3, 20130010.

[47] Wilkie, K., Hahnfeldt, P., 2013. Tumorimmune dynamics regulated in the microenvironment inform the transient nature of immune-induced tumor dormancy. Cancer Res. 3, 3534-3544.

[48] Wodarz, D., 2001. Viruses as antitumor weapons: defining conditions for tumor remission. Cancer Res. 61, 3501-3507.

[49] Wodarz, D., 2006. Killer Cell Dynamics: Mathematical and Computational Approaches to Immunology. Springer.

[50] Wodarz, D., Hofacre, A., Lau, J. W., Sun, Z., Fan, H., Komarova, N. L., 2012. Complex spatial dynamics of oncolytic viruses in vitro: mathematical and experimental approaches. PLoS Comput. Biol. 8 (6), e1002547. 
579

[51] Wodarz, D., Komarova, N., 2009. Towards predictive computational models of oncolytic virus therapy: basis for experimental validation and model selection. PLoS One 4 (1), e4271.

[52] Wu, J., Kirn, D., Wein, L., 2004. Analysis of a three-way race between tumor growth, a replication-competent virus and an immune response. Bull. Math. Biol. 66 (4), 605-625.

[53] Zhang, P., Côtè, A. L., de Vries, V. C., Usherwood, E. J., Turk, M. J., 2007. Induction of postsurgical tumor immunity and t-cell memory by a poorly immunogenic tumor. Cancer Res. 67, 6468-6477. 